\title{
Characterization of Ventricular Tachycardia and Fibrillation Using Semantic Mining
}

\author{
Mohd Afzan Othman ${ }^{1}$, Norlaili Mat Safri ${ }^{1}$, Ismawati Abdul Ghani ${ }^{1}$ \& Fauzan Khairi Che Harun ${ }^{1}$ \\ ${ }^{1}$ Dept. of Electronics Eng., Faculty of Electrical Eng., Universiti Teknologi Malaysia, Johor 81310, Malaysia \\ Correspondence: Norlaili Mat Safri, Dept. of Electronics Eng., Faculty of Electrical Eng., Universiti Teknologi \\ Malaysia, Johor 81310, Malaysia. Tel: 60-755-352-54. E-mail: norlaili@fke.utm.my
}

Received: May 31, 2012 Accepted: July 9, 2012 Online Published: July 18, 2012

doi:10.5539/cis.v5n5p35 URL: http://dx.doi.org/10.5539/cis.v5n5p35

\begin{abstract}
Ventricular tachycardia (VT) and ventricular fibrillation (VF) are potentially life-threatening forms of cardiac arrhythmia. Fast and accurate detection of these conditions can save lives. We used semantic mining to characterize VT and VF episodes by extracting three significant parameters (frequency, damping coefficient and input signal) from electrocardiogram (ECG) signal. This method was used to analyze four-second ECG signals from a widely recognized database at the Massachusetts Institute of Technology (MIT). The method achieved a high sensitivity and specificity of $96.7 \%$ and $98.3 \%$, respectively, and was capable of detecting normal sinus rhythm (N) from VT and VF signals without false detection, with a sensitivity of $100 \%$. VT and VF signals were recognized from each other, with a recognition sensitivity of $96 \%$ and $94 \%$, respectively. This newly proposed method using semantic mining shows strong potential for clinical applications because it is able to recognize VT and VF signals with higher accuracy and faster recognition times compare to existing methods.
\end{abstract}

Keywords: ventricular fibrillation, ventricular tachycardia, ecg, ventiruclar arrhythmias, life threatening arrhythmias, semantic mining

\section{Introduction}

Ventricular fibrillation (VF) is a fatal form of arrhythmia that is considered to be life threatening. VF is potrayed by irregular contractions of the heart's ventricles, resulting in a failure to pump blood and death within minutes unless corrective measures are taken promptly. An electric defibrillator can be used to return the heart to the normal sinus rhythm (N). However, if the combination of a normal sinus rhythm and ventricular tachycardia (VT) is misinterpreted as a false positive (FP) for VF, the patient receives a needless shock that could deteriorate the heart function and cause serious consequences to the patient. Conversely, an incorrect diagnosis for VF can result in a false negative (FN) and is life threatening. Therefore, the accurate and early detection of VT or VF is extremely important.

Automated systems for the detection of VF using various detection methods, such as time- and frequency-domain analysis, neural networks, wavelet and nonlinear analysis, have been described previously by other researchers. These automated systems can recognize the VF signal from either a NSR or a VT signal. Most of these methods used a time-domain analysis approach because of the advantage of using simple algorithms and real-time analysis via a computer. The time-domain analysis includes an autocorrelation function (AFC) (Chen et al., 1987; Clayton et al., 1993), a threshold-crossing interval (TCI) (Clayton et al., 1993), time-delay methods (Amann et al., 2007), and threshold-crossing sample counts (TCSC) (Arafat et al., 2009). Frequency-domain analysis is usually performed using power spectrum analysis, whereby the VF signal is characterized by observing the power spectrum of the signal (the VF power signal is reported to vary from 4 to $7 \mathrm{~Hz}$ ) (Clayton et al., 1993). Other methods using frequency-domain analysis include modified amplitude distribution analysis (MADA) (Fokkenrood et al., 2007) and multifractal singularity spectrum analysis (Wang et al., 2007).

When artifial neural network (ANN) techniques have been proliferated in late 1990's, most researchers opt for this technique because it has the ability to deal with nonlinear discrimination between classes (Clayton et al., 1994; Minami et al., 1999). In addition, wavelet transforms (Khadra et al., 1997; Abbas et al., 2004; Nawarvar et al., 2004) and nonlinear analysis (Jekova et al., 2002; Sun et al., 2005; Daoming et al., 2007) were proposed as detection techniques for the classification of VF signals. 
Despite the previous proposed methods shown promising results in terms of sensitivity and specificity, a few drawbacks have been identified to improve the accuracy of detection. Some of the proposed techniques are too complicated to be materialized in real time. For the discrimination of VT and VF signals, the detection time becomes the key factor to determine the patient's fate. According to Minami et al. 1999, the time delay due to the detection of VT/VF signals must be as short as possible; otherwise, the patient is at risk of death. If the proposed technique is too complex, the processing time will be too long to be effective. Additionally, some of the previous methods only discriminate between N and VF signals, without testing for VT (Amann et al., 2007; Clayton et al., 1994; Jekova et al., 2002; Arafat et al., 2009). As mentioned above, VF must be accurately identified and discriminated from VT and N signals to prevent any unnecessary shock to the patient that could damage the heart. The accuracy of the detection technique must be as high as possible to prevent misinterpretation or a false negative, which would have fatal consequences for the patient.

Previously, we have proposed a semantic mining technique that can characterize ventricular arrhythmia (V) from normal sinus rhythm (Othman et al., 2010; Othman et al., 2012). However, the previous study only concentrated on discriminating between $\mathrm{N}$ and $\mathrm{V}$. Because of this reason, in this study, we expand the technique instead of only characterize V, we try to characterize VT and VF as well. This method is essentially different from other approaches because this algorithm can portray the heart's oscillatory behavior by classifying behavior patterns using a semantic concept. This algorithm extracts the significant characteristics of the ECG signal (the frequency, damping coefficient and input signal) and classifies them into three types of rhythms: normal sinus rhythm (N), ventricular tachycardia (VT) and ventricular fibrillation (VF).

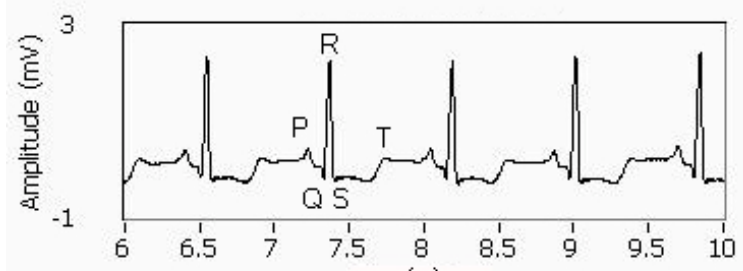

(a)

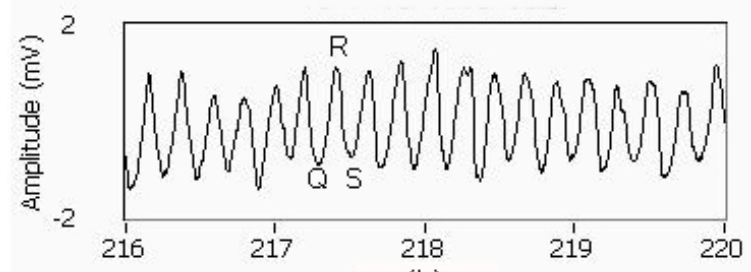

(b)

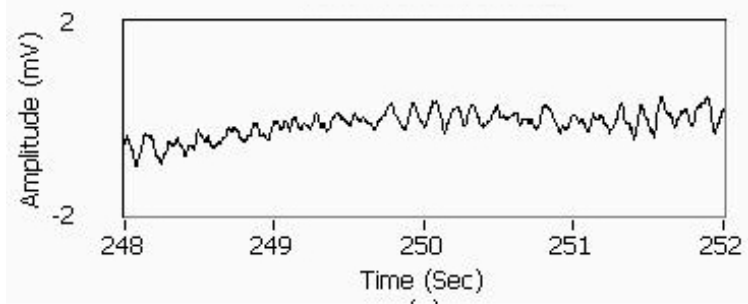

(c)

Figure 1. Examples of the ECG signals used: (a) Typical normal sinus rhythm (N) waveform; (b) typical ventricular tachycardia (VT) waveform; (c) typical ventricular fibrillation (VF) waveform

\section{Method}

\subsection{ECG Data}

The ECG data were acquired from the PhysioBank database (Goldbeger et al., 2000), which is a large and growing online database of well-characterized digital physiological signals and related data for use by the biomedical research community. In this study, the MIT 'nsrdb' (normal sinus rhythm database) with ECG recordings of people that only contains recordings of normal sinus rhythms and the MIT 'cudb' (The Creighton University ventricular tachyarrhythmia database) with ECG recordings of patients with sustained ventricular tachycardia and ventricular fibrillation were used. Figure 1 shows an example of the signal epochs used. The 
ECG signal for a normal sinus rhythm consists of identical PQRST waves. Typically, in a normal sinus rhythm ECG (Figure 1a), each P wave is followed by a QRS complex and then a T wave, and the rhythm is regular. In a ventricular tachycardia ECG (Figure 1b), no P waves are found, and the QRS complexes are wide and abnormal, with a rhythm that is sometimes regular and sometimes irregular. In Figure 1c, an ECG of ventricular fibrillation is shown; P waves and QRS complexes are absent, and the rhythm is chaotic.

\subsection{ECG Filtering and Processing}

Usually, a raw ECG signal contains noise, which affects the ability of certain ECG recognition systems to recognize patterns in ECG signals (Arafat et al., 2009; Jekova et al., 2002; Pan \& Tomkins, 1985). Examples of noise includes electrode motion artifacts, baseline wander and power line interference. Thus, a bandpass Butterworth was applied to ECG signals in order to reduce noise. A pass band from 1-30 Hz was chosen because the power spectrum of N, VT and VF signals are reported to be within this range (Chen et al., 1987; Clayton et al., 1993; Minamin et al., 1999).

The transfer function of second order Butterworth bandpass filter is

$$
H(z)=\frac{0.027+0.109 z^{-1}+0.164 z^{-2}+0.109 z^{-3}+0.027 z^{-4}}{1-2.791 z^{-1}+4.327 z^{-2}-2.791 z^{-3}+z^{-4}}
$$

After filtering, the resulting ECG signals were Fourier transformed using fast Fourier transform (FFT).

\subsection{Semantic Miniing}

A reduce-order agent model called semantic mining is used to portray the system's oscillatory behavior by monitoring it behavior pattern. It allows us to determine the nature of their behavior by determining the exact parameters that influences such behavior by only analysis its output (Al-Dabbas et al., 2004). Figure 2 illustrates the overall diagram of semantic mining system. In this study, the semantic mining algorithm was employed to extract significant parameters from ECG. These parameters (frequency, damping coffiecient and input signal) represent the oscillation behavior of the cardiovascular system. Equation 2 shows their interrelationships

$$
\omega^{-2} \cdot x^{\prime \prime}+2 . \zeta \cdot \omega^{-1} \cdot x^{\prime}+x=u \quad x(0)=x_{0} \quad x^{\prime}(0)=x^{\prime}{ }_{0}
$$

where $\omega$ is the natural frequency, $\zeta$ is the damping coefficient, $u$ the input and $x$ is the output of the ECG signal. The three syntax parameters $\omega, \zeta$ and $u$ may be constants, variables or may vary with dynamical behavior (Al-Dabbas et al., 2004). Three algorithms have been proposed by David Al-Dabass et al., 2004 for pattern recognition architecture. In this study, we opted to use algorithm three because it better predicted $\omega$ and $u$ when the extracted parameters were variable and when the system has second-order dynamics.

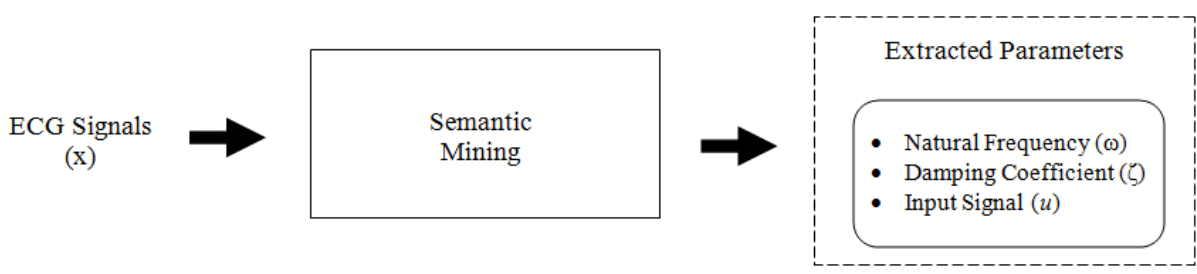

Figure 2. Overview of the semantic mining system

The algorithm uses fourth time derivatives at single point, denoted as $x$ ', $x$ ”, $x$,' and $x$,'”. The second-order system as follows;

$$
\omega^{-2} \cdot x^{\prime \prime}+2 \cdot \zeta \cdot \omega^{-1} \cdot x^{\prime}+x=u
$$

Differentiate with respect to $t$;

$$
\omega^{-2} \cdot x^{\prime \prime \prime}+2 \cdot \zeta \cdot \omega^{-1} \cdot x^{\prime \prime}+x^{\prime}=0
$$

divided by $x "$;

$$
\frac{\omega^{-2} \cdot x^{\prime \prime \prime}}{x^{\prime \prime}}+2 \cdot \zeta \cdot \omega^{-1}+\frac{x \prime}{x^{\prime \prime}}=0
$$

differentiate with respect to $t$ again; 


$$
\frac{\omega^{-2} \cdot\left(x^{\prime \prime} \cdot x^{\prime \prime \prime \prime}-x^{\prime \prime \prime} \cdot x^{\prime \prime \prime}\right)}{x^{\prime \prime 2}}+0+\frac{x^{\prime \prime} \cdot x^{\prime \prime}-x^{\prime} \cdot x^{\prime \prime \prime}}{x^{\prime \prime 2}}=0
$$

Therefore the expression for $\omega, \zeta$ using equation (3), and $u$ are:

$$
\begin{gathered}
\omega^{-2}=\frac{x^{\prime \prime} \cdot x^{\prime \prime \prime \prime}-x^{\prime \prime \prime 2}}{x^{\prime} \cdot x^{\prime \prime \prime}-x^{\prime \prime 2}} \\
\zeta=-\left(\frac{\omega^{-2} \cdot x^{\prime \prime \prime}+x^{\prime}}{2 \cdot \omega^{-1} \cdot x^{\prime \prime}}\right) \\
u=\omega^{-2} \cdot x^{\prime \prime}+2 \cdot \zeta \cdot \omega^{-1} \cdot x^{\prime}+x
\end{gathered}
$$

These three parameters- $\omega, \zeta$ and $u$-were extracted from the Fourier-transformed ECG signals and are required for further analysis. By using these extracted parameters (obtained from equations 7-9), the mean amplitude from each parameter is calculated. These mean amplitude can be analyzed and compared and is consequently useful in the detection of VT and VF signals.
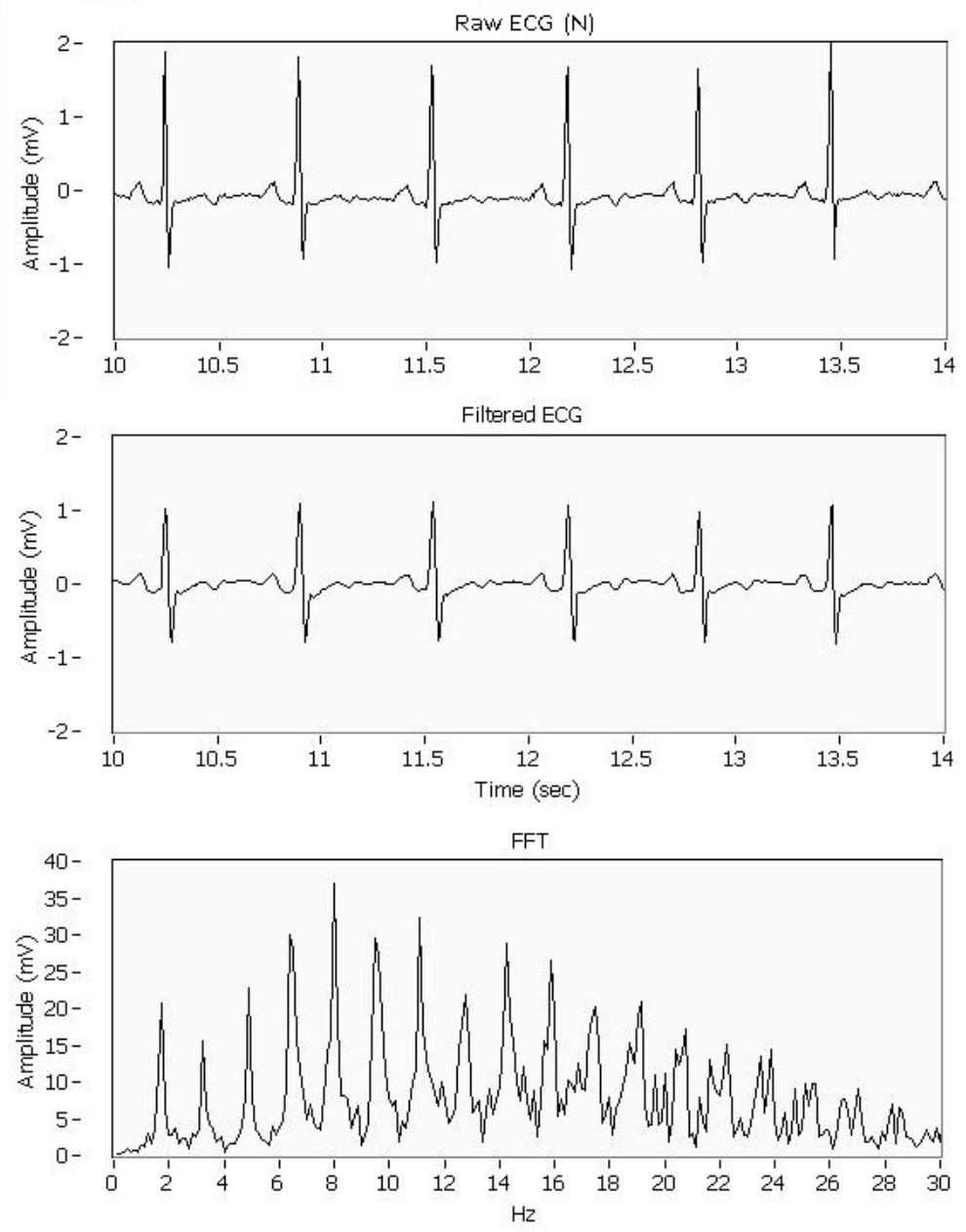

Figure 3. Illustration of the steps involved in the semantic mining algorithm for the analysis of $\mathrm{N}$ episodes: (a) Original ECG signal; (b) ECG signal after noise filtering; (c) Fourier-transformed ECG signal

\section{Results}

\subsection{Data Analysis}

Ten different ECG recordings of normal sinus rhythm, of ventricular tachycardia and of ventricular fibrillation were selected from the MIT nsrdb and MIT cudb. All records were from different person. For each of ECG record, we took five episodes to make a total of fifty ECG episodes per ECG type, i.e., fifty episodes of normal 
sinus rhythms $(\mathrm{N})$ were taken from the MIT nsrdb, while fifty episodes of ventricular tachycardia signals (VT) and fifty episodes of ventricular fibrillation signals (VF) were taken from the MIT cudb. Selection of these episodes was performed using annotations from a cardiologist provided in the database. Each episode was four seconds long.

Semantic mining was performed, and the three significant parameters $(\omega, \zeta$ and $u$ ) were extracted with equations 7-9. The mean amplitude extracted from each parameter was analyzed to find significant differences among $\mathrm{N}$, VT and VF episodes. Figures 3-5 show the process flow for each type of ECG episode. The raw ECG signal was sent to a preprocessing stage to reduce noise. After filtering noise, the resulting ECG signals were Fourier transformed, and these Fourier transform of ECG signals were used with the semantic mining technique to obtain the three significant parameters $\omega, \zeta$ and $u$. Then, the mean amplitude for each parameter was calculated.
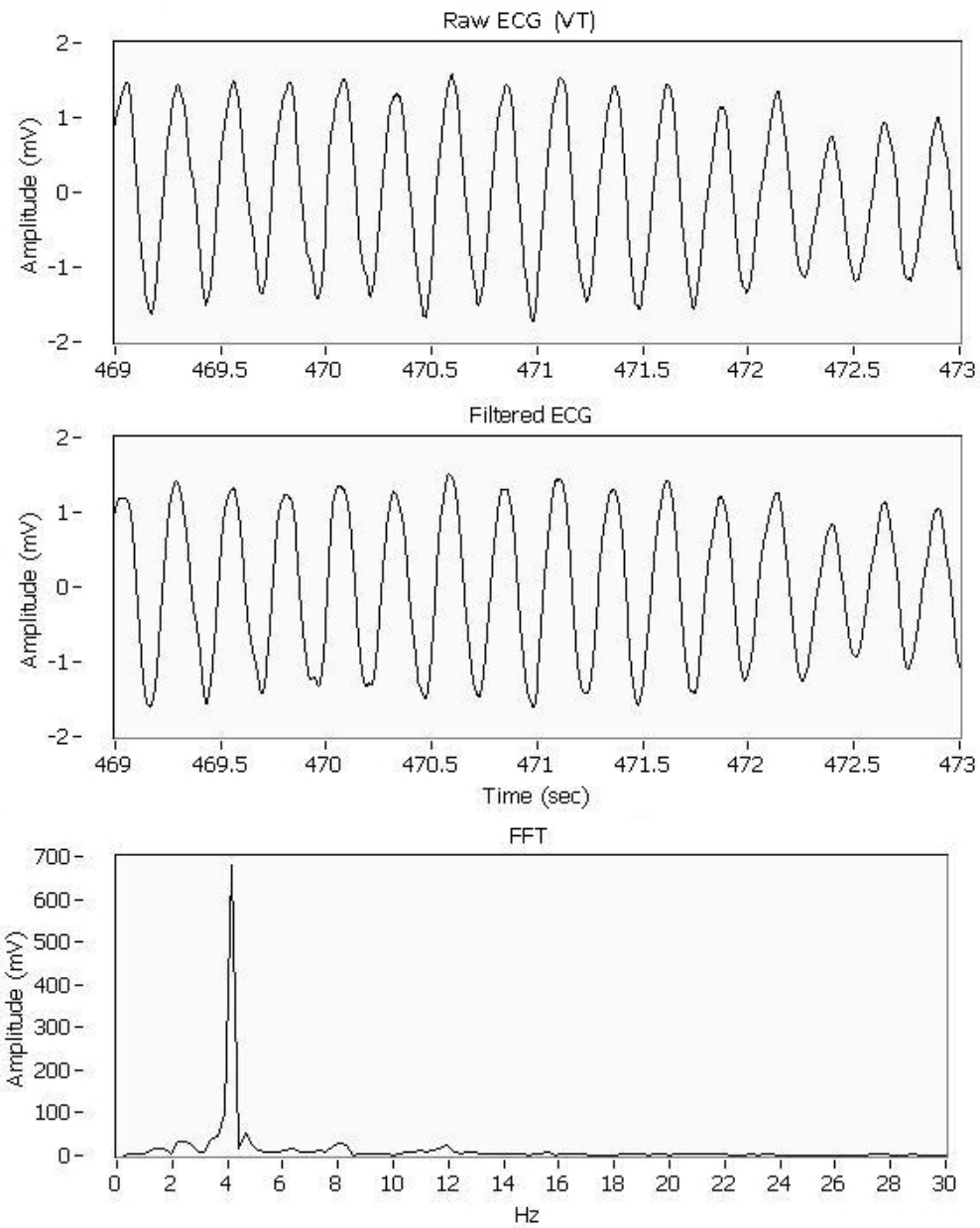

Figure 4. Illustration of the steps involved in the semantic mining algorithm for the analysis of VT episodes: (a)

Original ECG signal; (b) ECG signal after noise filtering; (c) Fourier-transformed ECG signal

An ANOVA with a single-factor model was adopted to preform the significance between the parameters extracted for N, VT and VF episodes. The ANOVA revealed that the mean amplitude and standard deviation for the natural frequency $(\omega)$ parameter and the input $(u)$ parameter showed extremely significant differences between $\mathrm{N}$, VT and VF episodes with $\mathrm{F}_{2,149}=1388.11$ and $\mathrm{P}<0.001$ and $\mathrm{F}_{2,149}=39.82$ and $\mathrm{P}<0.001$. However, ANOVA revealed no significant difference in the damping coefficient $(\zeta)$ for N, VT and VF episodes $\left(\mathrm{F}_{2,149}=\right.$ $0.71, \mathrm{P}=0.494)$.

Based on ANOVA, we considered only $\omega$ and $u$ for a T-test analysis to assess which two types of ECG episodes were statistically different from each other. It is apparent from Figure 6 that the $\omega$ values for N, VT and VF are distributed around the mean amplitude. The mean amplitude for $\mathrm{N}$ episodes has tendancy to be greater than the mean amplitudes for VT and VF episodes. With an upper threshold $\left(\mathrm{t}_{1}\right)$ set at 0.75 , as presented in Figure 6 , it is clear that the data from the $\mathrm{N}$ episodes are distinct from the data from the VT and VF episodes and that the mean 
amplitude of episodes larger than $t_{1}$ can be categorized as $\mathrm{N}$. At the same time, the data from the VT and VF episodes show that these two groups are separate from one another. In this case, a lower threshold $\left(t_{2}\right)$ was set at 0.58 , and episodes with a mean amplitude greater than $t_{2}$ but lower than $t_{1}$ fall into the VT group, while episodes with a mean amplitude smaller than $t_{2}$ fall into the VF group. This distinction is validated by a statistical analysis using a T-test in which the $\omega$ parameters from all three types of ECG episodes were shown to be statistically different from each other, as seen in Table 1.
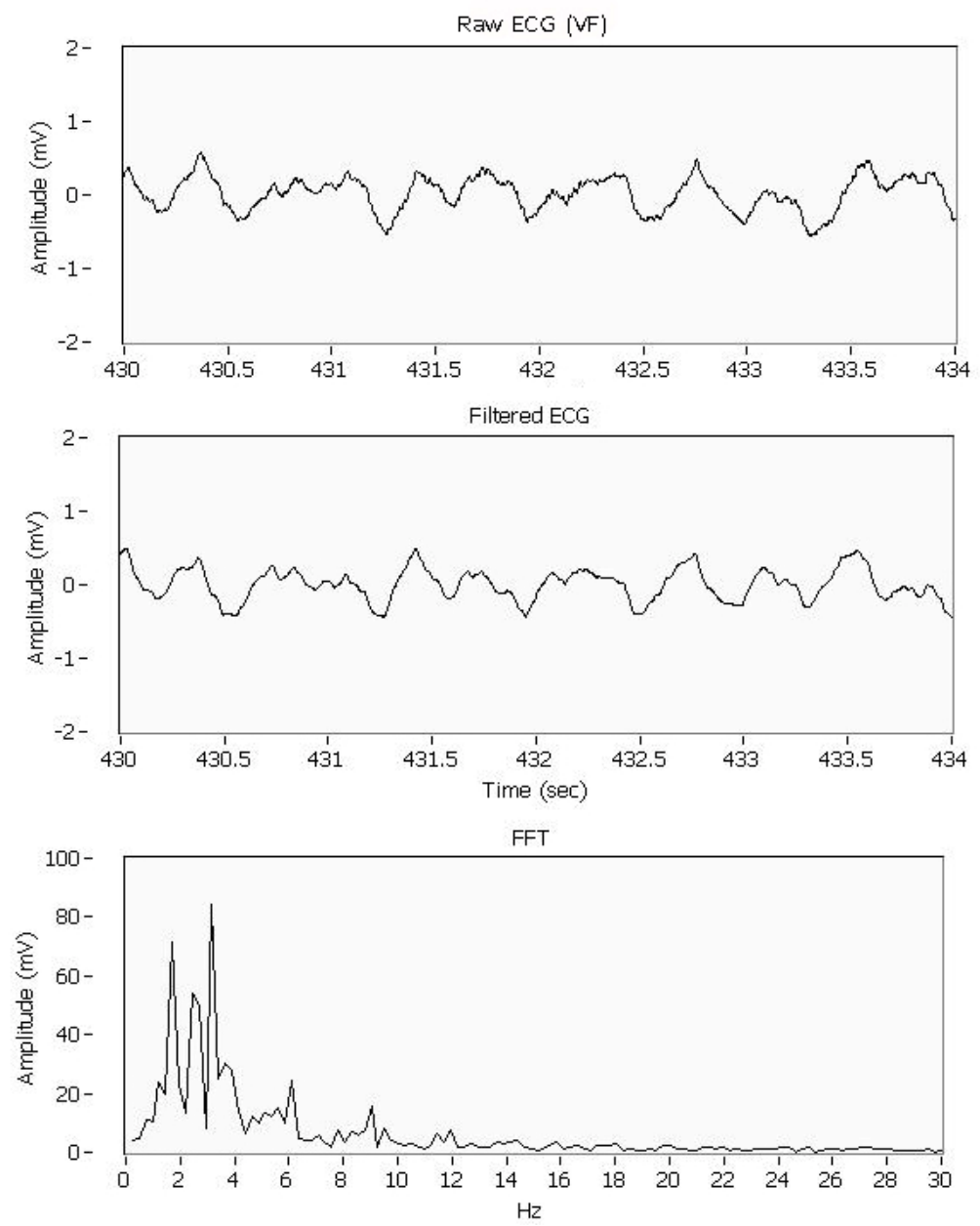

Figure 5. Illustration of the steps involved in the semantic mining algorithm for the analysis of VF episodes: (a)

Original ECG signal; (b) ECG signal after noise filtering; (c) Fourier-transformed ECG signal

Table 1. Mean amplitude distribution of ECG signal types

\begin{tabular}{cccc}
\hline \multirow{2}{*}{ Extracted Parameters } & \multicolumn{3}{c}{ Mean amplitude distribution of ECG types } \\
\cline { 2 - 4 } & $\mathrm{N}$ & $\mathrm{VT}$ & $\mathrm{VF}$ \\
\hline Natural Frequency $(\omega)$ & $0.905 \pm 0.006$ & $0.617 \pm 0.005$ & $0.553 \pm 0.004$ \\
Damping Coefficient $(\zeta)$ & $-0.003 \pm 0.003$ & $-0.004 \pm 0.002$ & $0 \pm 0$ \\
Input Signal $(u)$ & $3.748 \pm 0.319$ & $5.66 \pm 0.307$ & $2.444 \pm 0.121$ \\
\hline
\end{tabular}

Note: Data are presented as the mean \pm SEM.

The T-test with a two-tailed distribution and unpaired samples was used. The difference in the mean amplitude of $\omega$ between $\mathrm{N}$ and VT episodes was found to be significant $(\mathrm{P}<0.001)$, by means of the mean amplitude of $\mathrm{N}$ episodes $(0.905 \pm 0.006)$ significantly higher than that the mean amplitude of VT episodes $(0.617 \pm 0.005)$. The difference in mean amplitude between $\mathrm{N}$ and VF episodes using the T-test was also found to be very significant $(\mathrm{P}<0.001)$, by means of the mean amplitude of $\mathrm{N}$ episodes $(0.905 \pm 0.006)$ significantly higher than that the 
mean amplitude of VF episodes (0.553 \pm 0.004$)$. In addition, a significant difference between VT and VF episodes was found $(\mathrm{P}<0.001)$ using a T-test analysis for the mean amplitude of VT and VF episodes of $0.617 \pm 0.005$ and $0.553 \pm 0.004$, respectively.

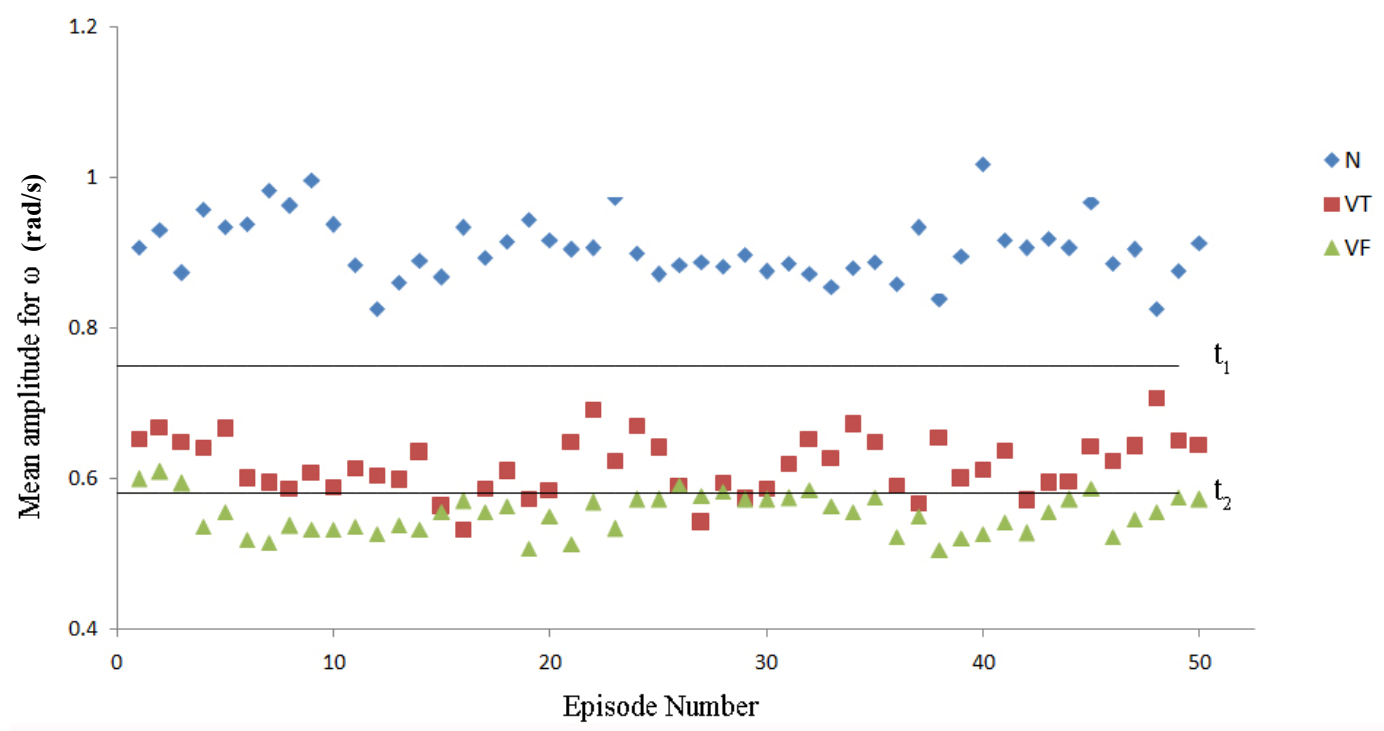

Figure 6. Mean amplitude distribution of the natural frequency $(\omega)$ parameter for N, VT and VF episodes The solid horizontal lines are the upper $\left(t_{1}=0.75\right)$ or lower $\left(t_{2}=0.58\right)$ thresholds.

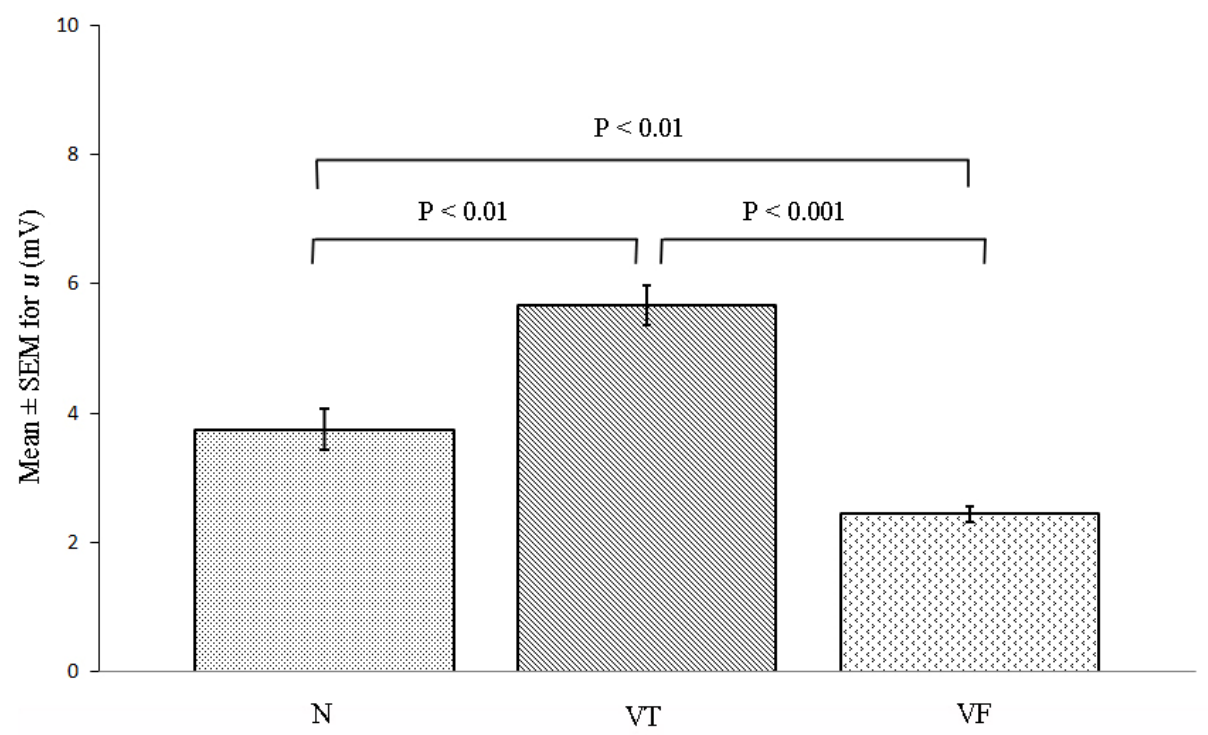

Figure 7. T-test analysis of the mean \pm SEM of the input $(u)$ parameter

According to the bar chart in Figure 7, we can concludes that, a significant difference in the mean $u$ value was found between VT and VF episodes $(\mathrm{P}<0.001)$, by means of the mean amplitude of the VT episodes $(5.66 \pm 0.307)$ notably higher than the mean amplitude of the VF episodes $(2.444 \pm 0.121)$. Although statistically significant differences were found between $\mathrm{N}$ and VT episodes and between $\mathrm{N}$ and VF episodes, the significance was smaller $(\mathrm{P}<0.01)$. It is apparent that some of the amplitudes of $\mathrm{N}$ episodes $(3.748 \pm 0.319)$ overlap with the mean amplitude of either VT or VF episodes. Table 1 summarizes the mean amplitude distribution for all types of ECG signal episodes.

\subsection{Data Comparison and Discussion}

To evaluate the performance of proposed algorithm, we calculate the specificity (Sp), sensitivity (Se) and positive predictive values (Pp) based on N, VT and VF detection. The Se, Sp and Pp were define as follows: 


$$
\begin{gathered}
\text { sensitivity }=\frac{T P}{T P+F N} \\
\text { specificity }=\frac{T N}{F P+T N} \\
\text { positivepredictive }=\frac{T P}{T P+F P}
\end{gathered}
$$

where TP is true positive (the signal detected and characterized correctly tested positive), TN is true negative (the signal detected and characterized correctly tested negative) and FP is false positive (the signal detected and characterized wrongly tested positive).

Table 2. Classification performance for different types of ECG signals

\begin{tabular}{ccccccc}
\hline ECG type & No. of episodes & TP & FP & FN & Se (\%) & Pp (\%) \\
\hline N & 50 & 50 & 0 & 0 & 100 & 100 \\
VT & 50 & 48 & 3 & 2 & 96 & 94.1 \\
VF & 50 & 47 & 2 & 3 & 94 & 95.9 \\
\hline Total & 150 & 145 & 5 & 5 & 96.7 & 96.7
\end{tabular}

Table 2 summarizes the performance of the semantic mining (SM) technique for the detection of N, VT and VF episodes from ECG signal data. A total of 150 episodes were tested to evaluate the performance of the technique, 50 episodes of which were type N, 50 episodes were type VT and 50 episodes were of type VF. There were no false positives for the detection of $\mathrm{N}$ type ECG episodes, with a sensitivity of $100 \%$. This means the VT and VF episodes can be distinguished completely from $\mathrm{N}$ episodes. For detection of VT and VF, two VT episodes were wrongly detected as VF episodes, while three VF episodes were wrongly detected as VT episodes. Thus it results the sensitivity of VT detection is $96 \%$ while VF detection is $94 \%$. However, the overall performance of the proposed algorithm is quite high, with a total sensitivity of $96.7 \%$.

Further investigation has been made to evaluate the performance of SM technique with other previously existing algorithms. We selected five existing techniques for comparison: phase-space reconstruction (PSR) (Amann et al., 2007), threshold-crossing sample counts (TCSC) (Arafat et al., 2009), modified amplitude distribution spectrum (MADA) (Fokkenrood et al., 2007), K-nearest neighbours rule (Jekova et al., 2002) and multifractal singularity spectrum (MSS) (Wang et al., 2007). These techniques yield promising results in their own right in terms of specificity and sensitivity.

Table 3. Comparison of the semantic mining algorithm with other methods

\begin{tabular}{ccccccc}
\hline Technique & PSR & TCSC & K-nearest & MADA & MSS & SM* $^{*}$ \\
\hline Sensitivity, \% & 83.8 & 92.9 & 95.2 & 97 & 99 & 96.7 \\
Specificity, \% & 95 & 95 & 95.7 & 98 & 99 & 98.3 \\
\hline
\end{tabular}

*: Proposed method.

Table 3 shows the sensitivity and specificity of the SM compared with other five that proposed by previous researcher. In this case, the sensitivity is defined as the ability to classify the ECG episodes correctly, and the specificity is defined as the ability to detect the absence of a characteristic in a signal without the characteristic. In other words, the specificity describes whether a VF episode was classified as being neither N nor VT. Among these methods, the MSS technique gave the highest sensitivity and specificity of $99 \%$, while the proposed SM technique and the MADA technique had only slightly lower sensitivity and specificity values. Although the sensitivity and specify of MSS was the highest, the duration of the ECG episodes used for MSS analysis were five seconds long compared with the analysis using the SM technique in which the ECG duration was only four seconds long. The analysis of data of longer duration can improve the accuracy of detection. However, because $\mathrm{VF}$ is considered a life-threatening arrhythmia and must be detected quickly, it is not always possible to take 
longer signals, and the analysis must be able to work efficiently with data from a short period of time.

As for analysis using the MADA technique, VF and VT episodes were not properly classified, which means that this technique cannot distinguish between the two. This is discouraging because implantable cardioverter defibrillators (ICDs) and automatic external defibrillators (AEDs) should be able to discriminate between VT and VF signals precisely. If VT is misread as VF, an unnecessary shock would be delivered, which could harm the heart; however, if VF is misread as VT, the low-energy cardioversion may not be sufficient to restore the heart to a normal sinus rhythm, which could be life threatening.

Our proposed method using semantic mining has a high sensitivity and specificity, which are close to those seen with the MSS method. Moreover, the episode duration used was only four seconds long, which is the shortest time period analyzed among the six tested methods (MSS was five seconds long, MADA was six seconds long, while PSR, TCSC and K-nearest were eight seconds long). Thus, the proposed SM algorithm has a shorter response time and higher accuracy in addition to good sensitivity and specificity.

\section{Conclusions}

A novel method for the detection of ventricular tachycardia and ventricular fibrillation has been presented in this paper. This method uses semantic mining to extract the significant characteristics of the ECG signal and classify them as N, VT or VF. The results show that the semantic mining method is capable of recognizing ECG patterns and can distinguish between N, VT and VF accurately. Moreover, the proposed method is superior in terms of detection time because the duration of the ECG episodes analyzed was shorter than those analyzed using existing methods. Taken together, the results show that the semantic mining algorithm is computationally efficient and well suited for real-time implementation.

\section{Acknowledgements}

The authors thank Siti Fatimah Mohamed Kamaruzzaman, M. D., who helped us with the medical aspects especially on interpreting the ECG signals. The research is financed by Universiti Teknologi Malaysia and MOHE through GUP Research Grant No. Q.J130000.7123.04J02.

\section{References}

Abbas, R., Aziz, W., \& Arif, M. (2004). Prediction of ventricular tachyarrhythmia in ecg using neuro-wavelet approach. National Conference on Emerging Technologies, Pakistan. pp. 82-87.

Al-Dabbas, D., Zreiba, A., Evans, D., \& Sivayognathan, S. (2004). Simulation of three parameter estimation algorithms for pattern recognition architecture. In Proceedings of $4^{\text {th }}$ UK Simulation Society Conference,7-9 April: Cambridge, England. 170-176. ISBN 0-905488-38-5.

Amann, A., Tratnig, R., \& Unterkofler, K. (2007). Detecting ventricular fibrillation by time-delay methods. IEEE Trans on Biomed Eng, 54, 174-177. http://dx.doi.org/10.1109/TBME.2006.880909

Arafat, M. A., Chowdhury, A. W., \& Hasan, M. K. (2009). A simple time domain algorithm for detection of ventricular fibrillation in electrocardiogram. Signal, Image \& Video Process, 5(1), 1-10. http://dx.doi.org/10.1007/s11760-099-0136-1

Arafat, M. A., Sieed, J., \& Hasan, M. K. (2009). Detection of ventricular fibrillation using empirical mode decomposition and bayes decision theory. Comput in Biol \& Med, 39, 1051-1057. http://dx.doi.org/10.1016/j.compbiomed.2009.08.007

Chen, S., Thakor, N. V., \& Mower, M. M., (1987). Ventricular fibrillation detection by a regression test on the autocorrelation function. Med \& Biol Eng \& Comput, 25, 241-249. http://dx.doi.org/10.1007/BF02447420

Clayton, R. H., Murray, A., \& Campbell, R. W. F. (1994). Recognition of ventricular fibrillation using neural networks. Med \& Biol Eng \& Computing, 32, 217-220. http://dx.doi.org/10.1007/BF02518922

Clayton, R. H., Murray, A., \& Campbell, R. W. F. (1993). Anderson, Comparison of four techniques for recognition of ventricular fibrillation from the surface ECG. Med \& Biol Eng \& Comput, 31, 111-117. http://dx.doi.org/10.1007/BF02446668

Daoming, Z., Guojan, T., Jifei, H., \& Yaofei, H. (2007). Ventricular arrhythmia nonlinear analysis. In Proceedings of International Conference on Intelligent Pervasive Computing, 11-13 October, Jeju Island, Korea. 57-61. http://dx.doi.org/10.1109/IPC.2007.37

Fokkenrood, S., Leijdekkers, P., \& Gay, V. (2007). Ventricular tachycardia/fibrillation detection algorithm for 24/7 personal wireless heart monitoring. In Proceedings of $5^{\text {th }}$ International Conference on Smart Homes and Health Telematics: June 2007: Nara, Japan. 110-120. 
Goldberger, A. L., Amara, L. A. N., Glass, L., Hausdorff, J. M., Ivanov, P. C., Mark, R. G., ... Stanley, H. E. (2000). PhysioBank, PhysioToolkit, and PhysioNet: Components of a New Research for Complex Physiologic Signals. Circulation, 101(23), 215-220. http://dx.doi.org/10.1161/01.CIR.101.23.e215

Jekova, I., Dushova, J., \& Popivanov, D. (2002). Method for ventricular fibrillation in the external electrocardiogram using nonlinear prediction. Institute of Physics Publishing, Physiological Measurement, 23, 337-345. http://dx.doi.org/10.1088/0967-3334/23/2/309

Khadra, L., Al-Fahoum, A. S., \& Al-Nashash, H. (1997). Detection of life threatening cardiac arrhythmias using wavelet transformation. Med \& Biol Eng \& Comput, 35, 626-632. http://dx.doi.org/10.1007/BF02510970

Minami, K., Nakajima, H., \& Toyoshima, T. (1999). Real-time discrimination of ventricular tachyarrhythmia with fourier-transform neural network. IEEE Trans on Biomed Eng, 46, 179-185. http://dx.doi.org/10.1109/10.740880

Namarvar, H. H., \& Sahidi, A. V. (2004). Cardiac arrhythmias predictive detection methods with wavelet-svd analysis and support vector machines. In Proceeding of $26^{\text {th }}$ IEEE Annual International Conference: 1-5 September, San Francisco. 365-368.

Othman, M. A., \& Safri, N. M. (2012). Characterization of ventricular arrhythmias using a semantic mining algorithm. Journal of Mechanics in Medicine \& Biology, 12(3), 307-311. http://dx.doi.org/10.1142/S0219519412004946

Othman, M. A., Safri, N. M., \& Sudirman, R. (2010). Characterization of ventricular arrhythmias in electrocardiogram signal using semantic mining algorithm. Mathematical/Analytical Modelling and Computer Simulation (AMS), 2010 Fourth Asia International Conference on 26-28May, 307-311.

Pan, J., \& Tomkins, W. J. (1985). A real-time QRS detection algorithm. IEEE Trans on Biomed Eng, 32, $230-235$. http://dx.doi.org/10.1109/TBME.1985.325532

Sun, Y., Chan, K. L., \& Krishnan, S. M. (2005). Life-threatening ventricular arrhythmia recognition by nonlinear descriptor. Biomed Eng Online. http://dx.doi.org/10.1186/1475-925X-4-6

Wang, G., Huang, H., Xie, H., Wang, Z., \& Hu, X. (2007). Multifractal analysis of ventricular fibrillation and $\begin{array}{llllll}\text { ventricular } \quad \text { tachycardia. } & \text { Med } & \text { Eng } & \text { Physics, } & 29, & \end{array}$ http://dx.doi.org/10.1016/j.medengphy.2006.05.007 